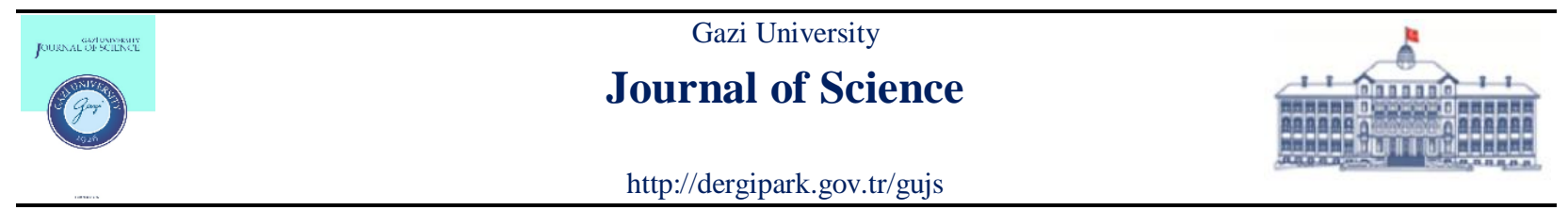

\title{
Energy Efficiency with Centralized Layering Approach for Multihop Wireless Sensor Networks
}

\author{
Mehmet SIMSEK ${ }^{*}$ (D), Sinan TOKLU (iD) \\ Department of Computer Engineering,Düzce University,81000, Düzce, Turkey
}

\author{
Highlights \\ - Improved network life time for wireless sensor networks. \\ - Improved throughput for wireless sensor networks \\ - Low protocol overhead. \\ - Multihop communication between sensor nodes. \\ - Layering and clustering sensor nodes.
}

\begin{tabular}{l} 
Article Info \\
\hline \\
Received: 16/04/2019 \\
Accepted: 27/05/2020 \\
Keywords \\
\hline Clustering \\
Sensor networks \\
Energy efficiency \\
Routing \\
Layering
\end{tabular}

\begin{abstract}
Sensors and Wireless Sensor Networks (WSN) are an important component of the Internet of Things (IoT) era. Sensors can be used in easily accessible areas such as factories; It is also used in places with difficult access, such as cultivated areas, forests etc. The most important constraint of sensors placed in areas where access is difficult is energy. Therefore, sensors need to spend as little energy as possible to communicate with each other. For this purpose, a number of studies were made concerning the optimization of energy in WSNs. Most of the studies are carried out in MAC and Routing Layers. In this study, we developed a new routing protocol called Base-Station Layered LEACH (BLLEACH) based on LEACH (Low Energy Adaptive Clustering Hierarchy) routing protocol, which is one of the most significant benchmarks in WSNs. BLEACH divides the network into clusters in a multi-hop environment. Experimental results show that BLLEACH outperforms LEACH and MDLEACH (Multi-hop dynamic clustering LEACH) in terms of energy efficiency and throughput.
\end{abstract}

\section{INTRODUCTION}

In recent years, WSNs have become an important area, which is widely used in phenomena such as technology advancement and easy access to and use of information that will facilitate human life. The nodes in the WSNs provide us with the opportunity to instantly obtain information such as temperature, humidity and motion in any desired location. Thus, it provides a technology that could be used in many fields such as inspection of military zones, disastrous regions, medical environments and locations with radioactive substances and that allows monitoring of and intervention into these fields in a more rapid manner [1]. Despite the fact that WSNs have many advantages, as the nodes use batteries as power sources, the power factor shortens the lifetime of the network, especially because of the nodes in hard-to-reach locations, and thus the communications breaks down. Naturally, the other nodes with power become inert and problems may be encountered in term of costing and communication. Hence, energy efficiency in WSNs is rather significant in this technology. Energy efficiency is provided in WSNs especially in two layers. These are MAC layer and Routing Layer [1,2]. As the subject of this paper is routing, studies made on routing layer were discussed.

Energy efficiency in WSNs is highly important for prolonging the lifetime of a routing network and ensuring uninterrupted communication. One of the purposes of using routing in WSNs is to overcome possible connection problems. In the literature, WSN routing is categorized in four different ways, which 
are network topology, communication models, topology-based and secure routing plans [2]. Some of the routing protocols that route in WSN assign equal and similar roles to all nodes in the network. On the other hand, in some routing protocols, each node may have a different role. A system that exists on other routing protocols dynamically controls the related values by checking the condition and energy levels of the network [3].

In protocols using network topology, the network architecture is used as the basis. When routing is carried out in WSNs, there may be differences in the connection of the nodes with each other, as well as the routes they follow during data communication from source to target. Here, the following topology is used: if Flat Protocol is used in the network, all the nodes in the same layer of the network have equal and similar roles. Flat protocols can be categorized as pro-active, reactive and hybrid protocols. All nodes that are part of the hierarchical protocol are divided into clusters within the network, and the node with highest energy becomes the $\mathrm{CH}$ (Cluster Head) of that cluster. CHs, however, manage activities inside and outside the cluster [4]. $\mathrm{CHs}$ are responsible for collecting data from nodes in their class, and they erase unnecessary data within the data collected without transmitting to the base station. Examples of this type of protocols are LEACH, SEP, TEEN and APTEEN. The distance between sensor nodes is calculated according to signal strength. A high signal strength indicates a short distance between the nodes. Some protocols here, allow nodes with no activity to switch to sleep mode [3,4].

\section{RELATED WORK}

Among routing protocol groups described in the introduction, Hierarchical Protocols include the most widely used protocols with respect to energy efficiency. Hierarchical protocols reduce the size of the routing table. In these protocols, nodes form the network over clusters. Each cluster is administered by the $\mathrm{CH}$ selected in each cycle. CHs periodically collect data in their own clusters, compress them and direct them to the base station. Each $\mathrm{CH}$ erases the duplicate data it receives in order to reduce the number of communications with the base station.

The LEACH protocol may be presented as the most basic routing protocol in WSN. In this protocol, data are collected at the cluster heads and directed to the base station to extend the lifetime of the network, to reduce the energy losses of the nodes, and to decrease the number of communication messages. In the protocol that uses clustering topology, member nodes within the cluster send their data to their own CHs. $\mathrm{CHs}$ summarize the data they receive and relay them to the base station. The disadvantages of LEACH are the uneven distribution of $\mathrm{CH}$ and that it runs in single-hop operation [4]. In another protocol, MODLEACH, the $\mathrm{CH}$ is not changed if the energy levels of the $\mathrm{CHs}$ in each cycle are not lower than the threshold value. In MODLEACH, the $\mathrm{CH}$ is changed when its energy level is lower than the threshold value. This, therefore, ensures energy saving by reducing the amount of energy consumed during cluster formation. MODLEACH divides communication into three categories: intra-cluster communication, intercluster communication, and data transfer from $\mathrm{CH}$ to base station. The energy required for communication within the cluster differs from the energy required for communication between clusters or from $\mathrm{CH}$ to the base station [5].

In another protocol, Stable Election Protocol (SEP), the nodes are divided into two types, which are normal nodes and advanced nodes. The initial energy levels of these nodes tend to vary. Advanced nodes perform the task of $\mathrm{CH}$ more often. This is because they have more energy compared to normal nodes [6]. Through central control algorithm, C-LEACH (Centralized LEACH) determines CHs within the network in a dispersed manner. This algorithm works on the base station and, the base station determines who will be the $\mathrm{CH}$. Using GPS, each node transmits its location and remaining energy level to the base station. The base station calculates an average energy level, and nodes with energy levels lower than the threshold value are not entitled to become $\mathrm{CH}$ for that particular cycle. Following the selection of the $\mathrm{CH}$, the base station broadcasts the message, containing the $\mathrm{CH}$ information of each node to all nodes in the network. Each node sends its own data to the $\mathrm{CH}$, then the $\mathrm{CH}$ performs data integration and sends it to the base station in a compressed file [7]. 
TL-LEACH (Two Levels LEACH) resolves the problem of unequal and unfair energy distribution in the LEACH protocol. This problem stems from the random selection of CHs. CHs may be selected randomly in advance and, for this reason, CHs may have different energy levels. This causes some nodes to die out early. In particular, CHs distant from the base station die out more quickly. To transfer the data collected at $\mathrm{CH}$ to the base station, the TL-LEACH uses secondary and primary $\mathrm{CHs}$. Secondary $\mathrm{CH}$ is responsible for collecting and integrating data from member nodes and transmitting them to the primary $\mathrm{CH}$. The primary $\mathrm{CH}$ is responsible for transmitting this data received from the secondary $\mathrm{CH}$ to the base station [8]. MH-LEACH (Multi Hop LEACH) protocol takes the LEACH protocol up an advanced level. It accomplishes this by using the nodes lying (extending) on the route to the base station. MH-LEACH follows the same path LEACH does in $\mathrm{CH}$ selection and cluster formation. However, instead of sending the $\mathrm{CH}$ data packet directly to the base station, MH-LEACH sends it to another $\mathrm{CH}$ on the route. Thus, the conservation of energy for $\mathrm{CHs}$ is ensured. In addition to this, in intra-cluster communication, the nodes send data packets to nearby neighboring nodes on the route instead of sending them directly to $\mathrm{CHs}$ and ensure energy conservation [9].

In this study, the LEACH protocol was improved through the energy-efficient multi-hop routing algorithm to extend the lifetime of the network and reduce energy consumption. Formation of clusters, data transfer and update of clustering stages in LEACH protocol were modified in the proposed algorithm. The proposed algorithm, to reduce energy consumption during the cluster formation stage, updates the Cluster Head and regulates the cluster topology. To reduce energy consumption during data transfer, it uses inter-cluster and intra-cluster multi-hop communication [10]. In another study carried out in this field, LEACH protocols and some newer versions were explained. Subsequently, an energy-efficient hierarchical routing protocol developed from LEACH protocol was proposed. As the basic concept here was to enhance energy efficiency, the study endeavoured to ensure radio communication in the shortest distance possible. New versions of the LEACH protocol adopted a heterogeneous multi-layer clustering approach. In this process, the distance between the base stations and the cluster heads was taken as basis. Three different types of nodes were used depending on the initial energy levels. Nodes had different roles depending on whether they were member nodes or cluster heads. It provided better energy efficiency compared to homogeneous LEACH protocols [11].

In another study conducted on multi-hop routing, Multi-hop-LEACH, a cluster-based routing protocol was proposed. The system proposed provides inter-cluster and intra-cluster multi-hop communication. This protocol was evaluated using TinyOs and TOSSIM simulation tools. When the evaluation results are considered, nodes consume less power for communication, and provide communication with less delay in this protocol. The connectivity, communication success rate and energy efficiency of the multi-hopLEACH protocol was further developed by increasing the probability of network clustering [12]. MRLEACH (Multi-hop Routing with Low Energy Adaptive Clustering Hierarchy) protocol divides the network into different layers to extend its lifetime. Cluster heads in each layer cooperate with neighboring layers to transmit data to the base station. Ordinary sensor nodes connect to the cluster heads via the Signal Strength Indicator (RSSI) received. The transmissions of nodes are controlled by the base station in accordance with the TDMA plan determined for each cluster head. The base station selects higher layer cluster heads so that they can act as super cluster heads for lower layer cluster heads. Thus, for energy conservation, MR-LEACH utilizes multi-hop routing from the cluster heads to the base station [13].

MDLEACH protocol takes dynamic clustering mechanism and multi-hop approach as basis. In fact, within the lifetime of the network, cluster heads change dynamically. Here, the process is divided into two cycles. At the beginning of each cycle, the number of cluster heads required is recalculated based on the surviving nodes in the network. MDLEACH adopts multi-hop approach for collecting and transferring data, as well. Here all the nodes are classified in three types, which are the Master-Heads (CMs), CHs and simple nodes (SNs). In the first hop, SNs transmit data to CHs. In the second hop, data collected from SNs are transmitted to CMs via CHs. In the third hop, CMs are responsible for collecting data and transferring to the base station [14].

Another protocol, PEGASIS, assumes that each node has global information about the position of the other nodes. Compared with other protocols, PEGASIS utilizes chain and dissemination topology for data 
collection. In this protocol, each node acts as receiver and transmitter for its nearby neighbor. That is, in every cycle, each node sends and receives a packet. PEGASIS demonstrates better performance than LEACH does. That is because it eliminates dynamic clustering, reduces transmission distance for non-CH nodes, and transfers data from the chain leader to the base station at once [15]. APTEEN (Adaptive threshold TEEN) and TEEN use two levels of clustering to send data to the base station. APTEEN is, basically, an advanced version of TEEN protocol that is designed for hybrid networks in particular. Here, CHs broadcast the following parameters to member nodes: Physical parameters, threshold values, TDMA plans, and maximum time between successive reports. APTEEN assists in the analysis of previous data values, monitors momentary incidents in the network and takes their snapshots [16]. In the TEEN (Threshold Sensitive Energy Efficient Sensor Network) method, sensors do not transfer data received to the $\mathrm{CHs}$ as received. The node transfers the data, only if the detected data is above a hard threshold value, or that the value detected is higher than or equal to the soft threshold value of a certain different parameter. This method reduces the number of data transfers. If the value detected is lower than the soft threshold value, then the transfer is not allowed. TEEN is more suitable for applications where data measurement is not continuous [17].

In three levels of hexagonal heterogeneous wide transmission distance protocol (3L - HEXA - HTBTDP) are introduced to increase the energy efficiency of the network. In this protocol, normal, enhanced and amplified sensor nodes in a hexagonal area represent three levels of heterogeneity. These nodes are selected as the cluster head (CHs) based on the new threshold values, so that the collected data is transferred to the base station. Scalability was performed to detect the differences of the proposed algorithm in the $3 \mathrm{~L}$ HEXA - HTBTDP application. For this, a different number of sensor nodes with variable values of initial energies were used. Different performance parameters such as live node measurement, stability period, network life, remaining energy and variance of energy were used for comparison [18].

In another work, contributes to the five input non-fuzzy equal clustering protocol (F5NUCP) to select the $\mathrm{CHs}$ to protect networks from the Hot Point problem and to determine the appropriate cluster size. Unequal clusters eliminate the hot spot problem by creating smaller clusters for nodes near BS and larger clusters for nodes far away from BS. F5NUCP uses an off-line approach to select temporary CHs by a back-off timer. Here, the timer value is set using the remaining energy of the node. The proposed method uses five input parameters. These are the remaining energy, distance to BS, distance to adjacent nodes, link quality and node degree. The output fuzzy parameters used in this study are the probability of being cluster size and $\mathrm{CH}[19]$.

In the case of LEACH-like studies, an advanced algorithm called ESO-LEACH has been proposed to provide solutions for issues such as the lack of cluster heads and ignoring the inexhaustible power of nodes. Here, metacognitive particle flock development is initially used to aggregate sensor nodes. To minimize the random nature of the algorithm in ESO-LEACH, the choice of advanced nodes and advanced rules is used in $\mathrm{CH}$ selection. Python based reproduction outputs are used for the operations [20].

In this study, the effectiveness of LEACH and LEACH based protocols in extending the life span of energy restricted WSNs was investigated. They have also proposed a LEACH clustering protocol called Advanced Multi-hop LEACH. This new protocol reduces and balances energy consumption to deliver more packet delivery and network life in WSNs. Here, the selection of the cluster head and new rules for calculation based on the remaining energy are proposed. Also, the multi-hop communication model is integrated into the WSN using two operating processes. These are levelling and general multi-hop routing [21].

Also, a new idea is presented to combine the selection mechanisms of nodes for energy efficiency in order to perform energy efficient routing and clustering operations in wireless sensor networks. In Wireless Sensor Networks, routing for nodes is a difficult and deceptive task. Also, Particle Swarm Optimization (PSO) was used in this study. In this way, a new energy efficient clustering scheme is also proposed for WSNs. The clustering algorithm is used to minimize the distance within the cluster simultaneously, in addition to the mentioned areas of use [22]. In [23], the centroid strategy was used. A new bat algorithm variant combined with this strategy is presented. In addition, three different centroid strategies are introduced. These strategies have six different designs. In addition, the speed inertial update equation is 
also used. The performance of the Optimization performed is confirmed by the CEC2013 criteria. WHCBA is integrated into the LEACH protocol. Thus, a two-stage cluster head node selection strategy has been developed.

In [24], a quasi dynamic clustering algorithm was proposed to reduce energy consumption and prolong the life of the network. The proximity of all nodes in the network to the closest gateways was calculated. Nodes are divided into two groups according to their proximity. The nodes in the first cluster according to the degree of proximity are assigned to the fixed gateway cluster. The nodes in the other cluster are dynamically correlated according to the current network statistics. In [25], a new clustering technique based on a genetic algorithm using the newly defined objective function is proposed. The proposed clustering method generally changes the steady state phase of the LEACH protocol in a heterogeneous environment. The proposed objective function evaluates three parameters in optimization, such as separation, compactness and number of cluster heads. Also in [26], common model control is recommended to meet the limitations in WSNs. A formal framework has been created to understand the proposed methodology, in particular to compare LEACH and extensions with LEACH-C and LEACH-F. The proposed approach has been shown to be effective in matters of security, liveness and accessibility.

Only a small number of the studies in the literature reviewed and discussed above offer solutions for multihop networks. These solutions are generally tested with small-scale scenarios in which sensor nodes can directly reach the base station. In the study, the BLLEACH was tested in a wide area and in scenarios where inter-node distances were relatively far.

\section{THE PROPOSED METHOD (BASE STATION LAYERED LEACH PROTOCOL}

The basic principle of BLLEACH is that the measured data is transferred in a way that it moves closer to the base station at each step. That is why the entire network is divided into layers instead of clusters. When the network is first set up, all nodes send their data to the base station over one another. Here, a graph neighboring table is created on the base station based on the data came from all nodes, and the layer of each node (the distance to the base station in hop count) is determined. The way BLLEACH works is shown in Algorithm 1.

Algorithm 1.

Step 1 All nodes send to the network a Hello message in which they attached their IDs.

Step 2 If a node receives a Hello message that does not contain its ID, it attaches its own ID to the message and sends it to the network.

Step 3 Base station waits for a certain amount of time for all the Hello messages to arrive and uses the contents of these messages to create the topology of the network.

Step 4 Base station divides the network into layers according to the hop count.

Step 5 Base station determines the number of Layer Gateways (LG) that must exist on each layer using Equation (1).

Step 6 Base station puts all nodes on each layer in order, based on the number of neighbors on the next layer, and creates an Ordered Nodes List (ONLL) for each Layer.

Step 7 Base station calculates how many LGs to change in every cycle using Equation (2).

Step 8 Base station creates an ONLL Announce (OA) packet, together with the ONLL, using information on the cycle duration, the number of LGs required on each layer, and number of LGs to change in each cycle, and then sends this packet to all nodes. 
Step 9 Nodes receiving the OA packet determine in which cycle they will become LG. The node that will become LG, announces itself by an LG announcement.

Step 10 Nodes receiving an LG announcement check which layer the announcement came from. If the announcement comes from the layer above, then the nodes send out Join Request message. If not, the nodes ignore the announcement.

Step 11 LG receiving a Join Request message adds the node sending the message in the Member List and sends out a Join Request Acknowledgement. Additionally, the LG node set up a TDMA schedule and sends the schedule to the member nodes.

Step 12 Communication starts.

Step 13 At the end of each cycle, the process is repeated starting from step 9.

Let us explain the algorithm on a sample topology.

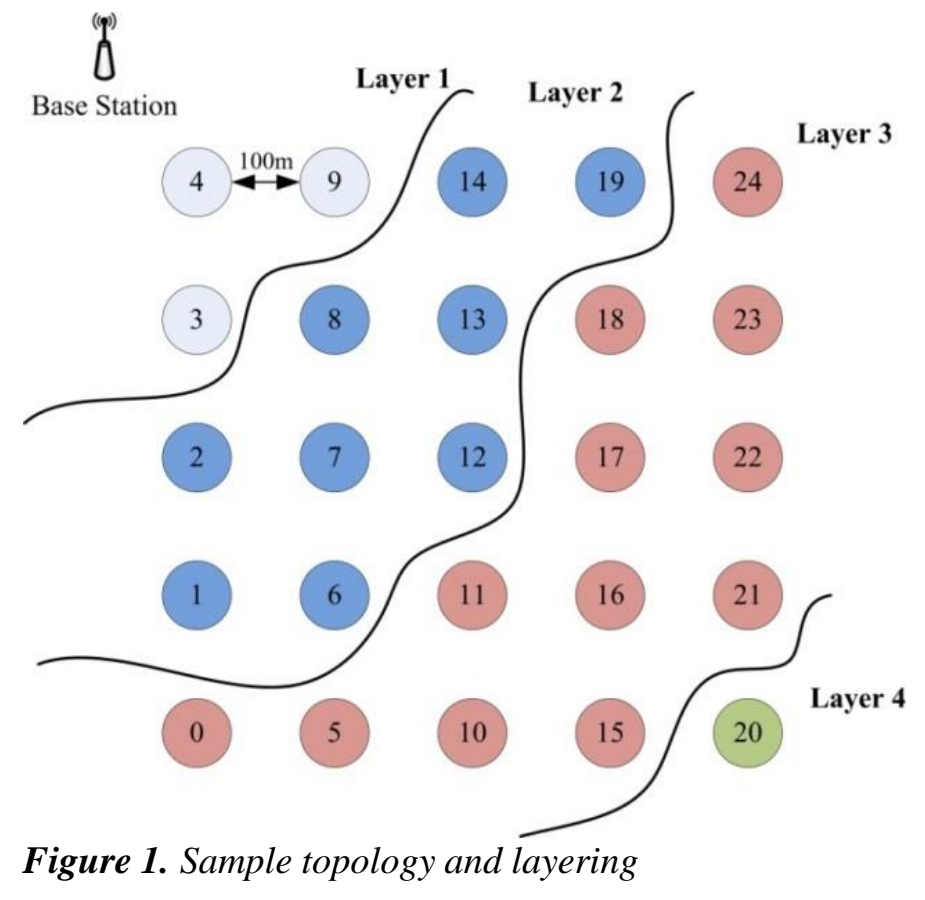

A sample topology with 25 nodes is shown in Figure 1. The base station divides the network into layers running the first four steps given in Algorithm 1. At this stage, an adjacency matrix of the network is created. In the sample topology, while the nodes in Layer 1 directly reach the base station, the nodes in Layer 3 reach the base station in 3 hops.

After this stage, the base station determines the number of Layer Gateways (LG) required for each layer using Equation (1).

$L G$ in a layer $=\left[\min \left(\left(\frac{n}{20} \times(\mu-\beta+1)\right), \gamma\right)\right\rceil$

where

$n$ : Number of nodes on layer below;

$\mu$ : Maximum hop count;

$\beta$ : Present layer;

$Y$ : Number of nodes in the present layer. If the result of the innermost parenthesis is higher than the value, the lower one is taken as the result. 
For example, the LG count in Layer 1 will be calculated as follows:

$L G$ in a layer $1=\left\lceil\min \left(\left(\frac{9}{20} \times(4-1+1)\right), 3\right)\right\rceil$

where, 9 is the total number of nodes in the layer below, which is Layer 2;

4 is the maximum hop count;

1 (written in bold) is the present layer, which is Layer 1;

3 is the total number of nodes on Layer 1 . We get 2 from the equation. As a result, there will be 2 LGs at any one time on Layer 1. Similarly, the number of LGs is calculated as 2 for Layer 2, and 1 for Layer 3. \# LG for Layer 4 cannot be determined, because there are no layers coming after it.

Subsequently, the base station puts all nodes on each layer in order based on the number of neighbors on the next layer, and creates an Ordered Nodes List for each Layer (ONLL). For instance, the node 3 on Layer 1 has 8 neighbors in Layer 2; the node 4 has 5 neighbors in Layer 2; the node 9 has 7 neighbors in Layer 2. These values may easily be calculated using the adjacency matrix in. Hence, the ONLL for Layer 1 is 3,9,4. ONLL is similarly formed for other layers. Subsequently, the base station calculates how many LGs will change in each cycle using Equation (2).

LGs to change $=\left|\min \left(L, \frac{K}{L}\right)\right|$

where, $L$ is the number of LGs required at any one time on a layer; $K$ is the number of non-LG nodes. For example, for Layer 1, the value of the number of LGs to change is calculated as 1. Base station then creates an ONLL Announce (OA) packet, together with the ONLL, using information on the cycle duration, the number of LGs required on each layer, and number of LGs to change in each cycle, and then sends this packet to all nodes. The OA packet for Layer 1 is shown in Figure 2.

\begin{tabular}{|c|c|c|c|} 
ONLL & Cycle Time & Number Of LGs & $\begin{array}{c}\text { Number Of LGs to } \\
\text { Change }\end{array}$ \\
\hline $3,9,4$ & 20 & 2 & 1 \\
\hline
\end{tabular}

Figure 2. OA packet structure

The nodes receiving the OA packet determine in which cycle they will become LG. The node to become LG announces itself by an LG announcement. For instance, when node 3 receives an OA packet as shown in Figure 2, it can see from here that it will be the LG in the first cycle, as it is at the top of the list. In addition, as 1 LG will change in each cycle, node 3 can calculate that after 20 seconds it will no longer be an LG, and act as a normal node. Similarly, node 4 can calculate that it is not an LG in the first cycle, but will be an LG in the following cycle, and that this will continue for 2 cycles. This calculation process continues in a loop until the node dies out. As the decrease in the energy levels of the nodes will be directly proportional to the duration they are an LG, the process of change of LGs was put in an order. In LEACH, $\mathrm{CH}$ changes are performed based on the energy levels of the nodes however, as it is not practical to calculate and sum up the remaining energy levels after each cycle this method was not adopted. This process may be likened to moving forward the window shown in Figure 3 in each cycle, in line with the time.

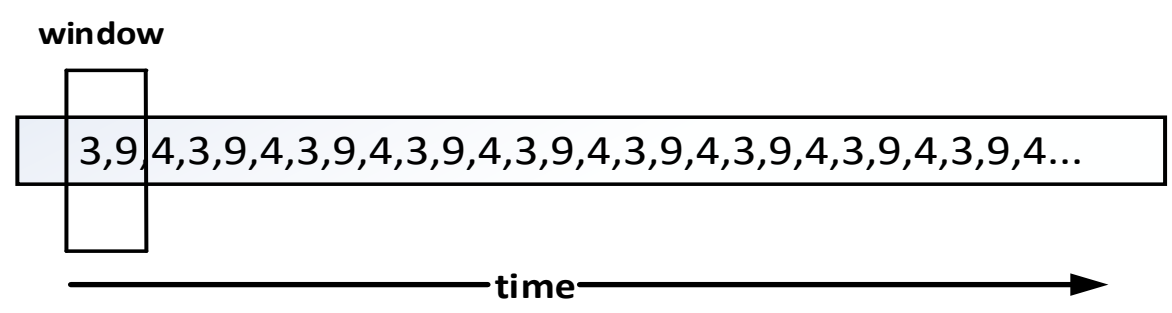


Figure 3. Deciding the LGs in a layer

The number of 'forward movements' is as many as the number of LGs required to change in each cycle. For Layer 1 in our sample, this value is 1, and the window moves forward one by one in each cycle. Later, the nodes receiving an LG announcement check which layer the announcement comes from. If the announcement comes from the layer above, then the nodes send out Join Request message. If not, the nodes ignore the announcement. For instance, if node 3 makes an LG announcement, the nodes numbered 1, 6, 2, 7 and 12 from Layer 2 may send a Join Request message. However, node 4, hearing the same announcement, ignores it because the announcement comes from a node on the same layer.

An LG receiving a Join Request message adds the node sending the message in its Member List and sends out a Join Request Acknowledgement. Additionally, the LG node sets up a TDMA schedule and sends the schedule to the member nodes. Here, the crucial point is how much time slot is to be allocated to each member. Proceeding from our sample, while the nodes numbered 1, 6, 2 and 7 in the member list of LG number 3 are initially normal nodes, the node 12 is an LG node for Layer 2. Therefore, when an LG node broadcasts a Join Request message it denotes in the message content that it is itself an LG. In this case, a longer time slot must be allocated to node 12 . This amount was empirically calculated twice as much as this amount. Figure 4 shows a sample TDMA schedule scheme.

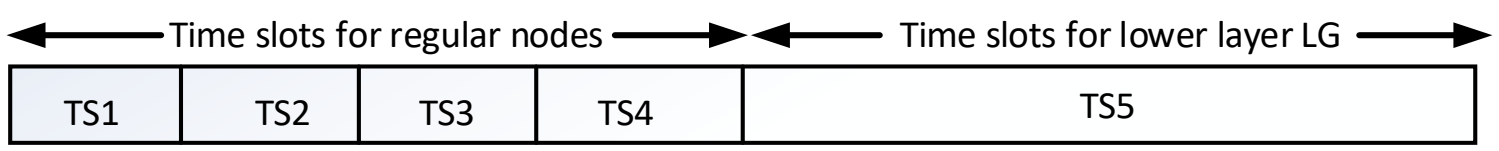

Figure 4. Sample TDMA schedule scheme

Following completion of all these stages, communication commences in the network. A sample communication instant is shown in Figure 5. The nodes numbered 3, 9, 12, 13 and 16 in Figure 5 are LGs.

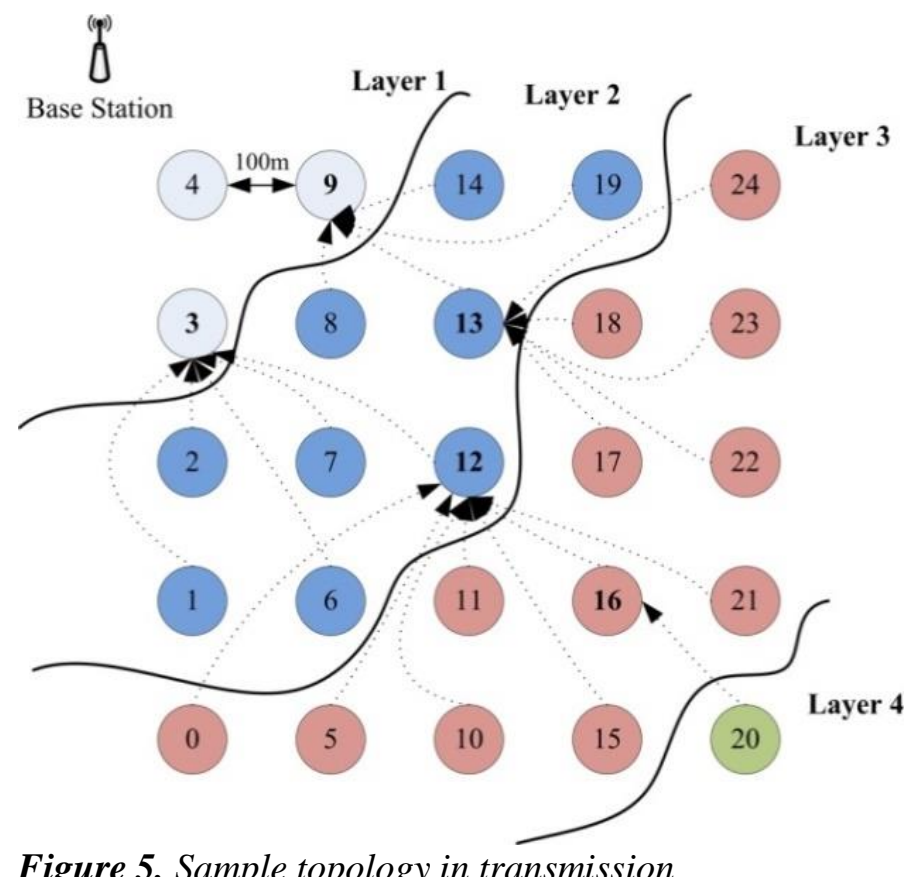

\section{EXPERIMENTAL RESULTS}

In order to test the performance of BLLEACH, a number of simulations were carried out on ns 2 simulator and it was compared to LEACH and MDLEACH protocols. The topology used in the simulations is shown in Figure 6. There are 100 sensor nodes and 1 base station in the simulations. Vertical and horizontal distances between the nodes were defined to be 100 meters. Even though the LEACH assumes that every node could send data directly to the base station, this is not always possible in large-scale applications. 
Therefore, maximum transmission range of each node (Excluding the base station) was limited to 210 meters in BLLEACH simulations. Parameters of the simulation are shown in Table 1. As the distance between the nodes quite long in the topology defined, nodes consume more energy per unit of data. The initial energy was set at $2 \mathrm{~J}$, as generally used in literature, and experiments were conducted. However, in order to get the simulation results for longer durations, the initial energy value was set at a higher value of $20 \mathrm{~J}$.<smiles>[CH][CH]</smiles>

Base Station

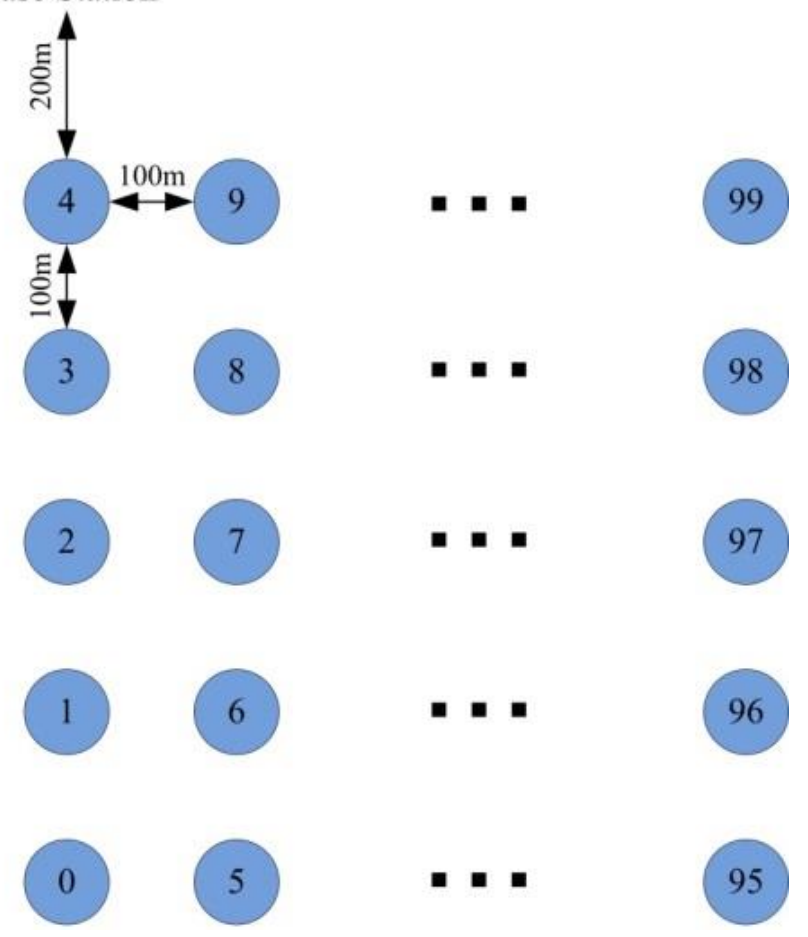

Figure 6. Simulation topology

Table 1. Simulation parameters

\begin{tabular}{|l|l|}
\hline Parameter & Value \\
\hline Network Size & $2000 \mathrm{~m} \times 1000 \mathrm{~m}$ \\
\hline Number of Sensor Nodes & 100 \\
\hline Initial Energy & $20 \mathrm{~J}$ \\
\hline Data Message Length & 500 byte \\
\hline Maximum Transmission Range & $210 \mathrm{~m}$ \\
\hline Simulation Time & $1000 \mathrm{~s}$ \\
\hline
\end{tabular}

The energy consumption values obtained from simulations are shown in Figure 7. In the simulation in which LEACH was used, the simulation was ended at the $900^{\text {th }}$ second as the number of nodes on the network became lower than the number of $\mathrm{CHs}$ required. Therefore, there are no values for LEACH protocol at the $1000^{\text {th }}$ second. BLLEACH consumed $17.53 \%$ less energy compared to LEACH and 13.13 $\%$ less energy compared to MDLEACH. 
The number of nodes survived in the simulations is shown in Figure 8. At the $900^{\text {th }}$ second, 3 nodes survived in the LEACH simulation. At the end of 1000 seconds, 64 nodes survived in BLLEACH simulation, and 44 nodes survived in MDLEACH simulation.

Finally, the amount of collected data is presented in Figure 9 for each protocol. BLLEACH transmitted to the base station $69.41 \%$ more data compared to LEACH, and $47.57 \%$ more data compared to MDLEACH.

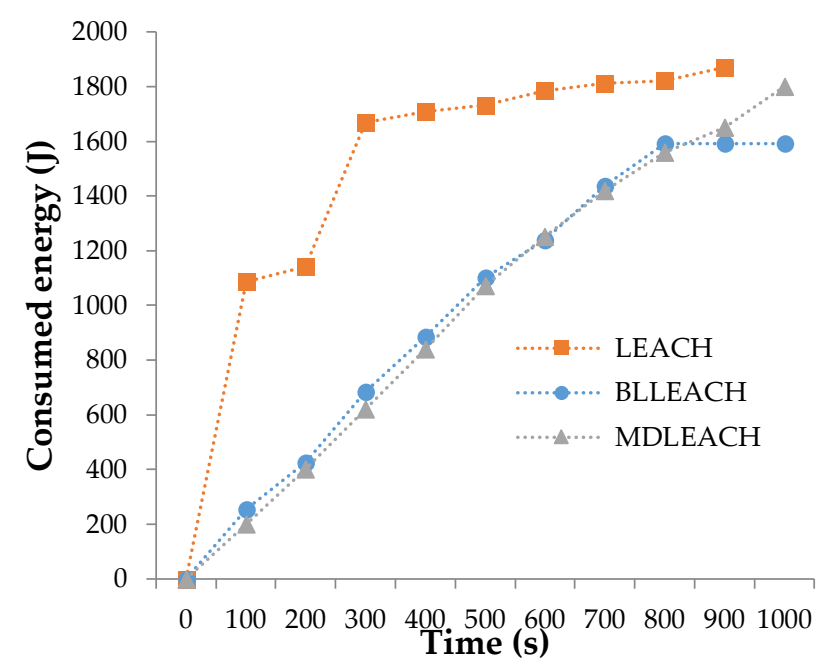

Figure 7. Energy consumption

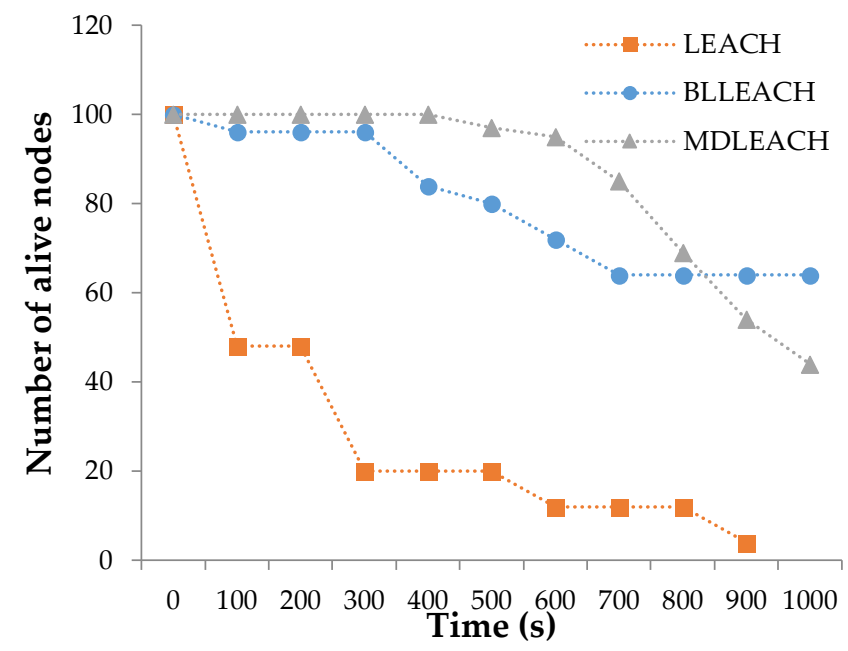

Figure 8. Network lifetime 


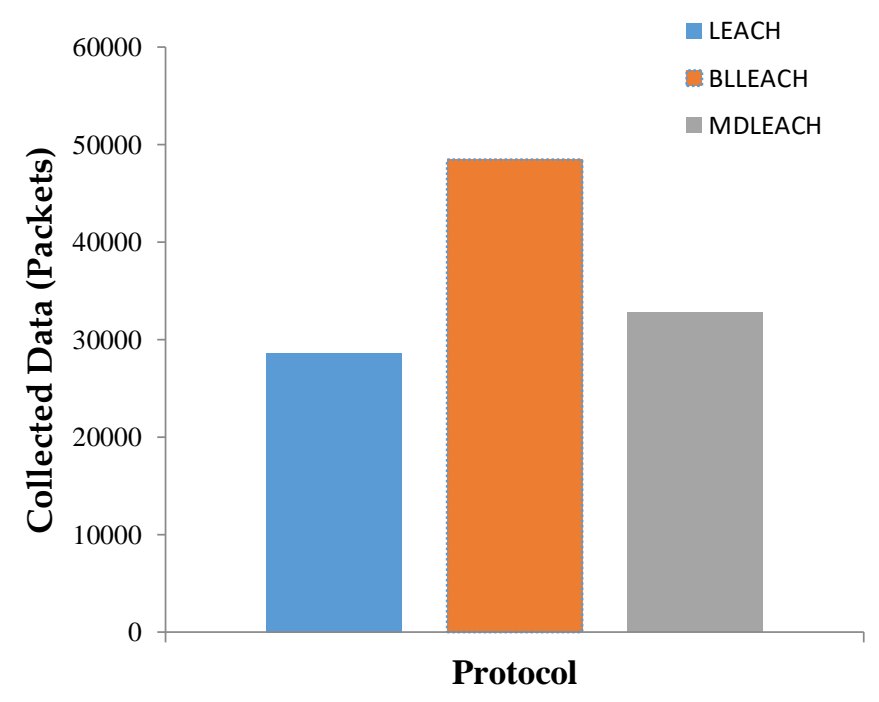

Figure 9. Total transmitted data

When the simulation results are evaluated together, BLLEACH provided more data to be collected by consuming less or the same amount of energy.

\section{DISCUSSION AND CONCLUSION}

The most critical limitation of wireless sensor networks is the insufficient energy. Reaching the sensor nodes at relatively inaccessible points and replacing their batteries, their energy sources, is associated with significant problems both, in terms of cost and the difficulty of accessing these locations. Therefore, to ensure a longer lifetime for the network, it is of the utmost importance to make efficient use of batteries that are the energy sources for the sensor nodes. In most applications, direct data transfer from the sensor nodes to the base station lowers energy efficiency. In large-scale applications, on the other hand, it is impossible for the sensor nodes to relay data directly to the base station. In such applications, it is necessary to use some nodes as hop points for other nodes. As the amount of data passing from nodes is higher on the nodes closer to the base station, energy consumption on these nodes is higher. As the death of these nodes means that the communication will be interrupted and the lifetime of the network will end even when there is energy on other nodes, nodes closer to the base station require better energy saving. In this study, LEACH protocol and its derivatives were briefly explained and their shortcomings were described. A new protocol was proposed to overcome these shortcomings and ensure a longer network life. BLLEACH divides the entire network into layers. Looking at the results obtained from our simulation, it was observed that BLLEACH is more successful than the other protocols.

However, BLLEACH also has certain limitations. One of these is scalability, which is a limitation of other protocols. In applications where the number of nodes is too high, the fact that the sensor nodes transmit packets in flooding to determine the network topology would result in energy loss. In addition, since the tasks the base station is required to fulfil increases, the setup time for the network would be extended. Another limitation is that nodes running out of energy are not removed from the ONLL. As the ONLL is created only once for each layer, it would lose its up-to-datedness over time. This leads to a situation where already-dead nodes are expected to become LGs. As a solution, the base station may recreate the network topology and the ONLL by demanding a HELLO message from all nodes in the network at certain periods (e.g. by predicting how long it will take certain number of nodes to die out). Even though this method provides a solution to the problem, it would also overload the network. Instead, a dispersed method may be developed. In addition, it would be beneficial to develop a practical approach that regulates the ONLL sequence taking into consideration the energy status of the nodes as the time goes by. Another limitation is the necessity that all nodes in the network are required to memorize the ONLL belonging to their layers. The ONLL created for networks consisting of several hundred of nodes would not be long lasting however, this is different for large networks. In this case, it is necessary to increase the memories of sensor nodes or 
in a better approach, to setup a more effective ONLL sharing mechanism. Another method might be to divide layers into sub-layers and create ONLLs for each of them.

It is possible to improve BLLEACH even further. For instance, when LGs in the layer below are determined, nodes in the layer above (the layer closer to the base station) that are neighbouring to more LGs may be selected. This will enhance the connectedness of the network, and delay the fragmentation time of the network. When TDMA schedule schemes are created, TDMA schemes of the other LGs may be created starting with the LGs closer to the base station. However, this is an optimization problem on its own. Another logic that was applied in the method developed is: "no need to shout when no one around to hear." In other words, when a node is not connected to an LG that will transmit its data to the base station, it will not try to transmit its data, thus it will conserve its energy. When a nearby node becomes an LG in time, it will restart transmitting data. Therefore, BLLEACH is a promising protocol, and one that is open to improvements.

\section{CONFLICTS OF INTEREST}

No conflict of interest was declared by the authors.

\section{REFERENCES}

[1] Akyildiz, I.F., Su, W., Sankarasubramaniam, Y., Cayirci, E.,"Wireless sensor networks: a survey”, Comput. Networks, 38(4): 393-422, (2002).

[2] Pantazis, N.A., Nikolidakis, S.A., Vergados, D.D., "Energy-Efficient Routing Protocols in Wireless Sensor Networks: A Survey", IEEE Commun. Surv. Tutorials, 15(2): 551-591, (2013).

[3] Arora, V.K., Sharma, V., Sachdeva, M., “A survey on LEACH and other's routing protocols in wireless sensor network", Optik (Stuttg).,127(16): 6590-6600, (2016).

[4] Heinzelman, W.B., Chandrakasan, A. P., Balakrishnan, H., "An application-specific protocol architecture for wireless microsensor networks", IEEE Trans. Wirel. Commun., 1(4): 660-670, (2002).

[5] Mahmood, D., Javaid, N., Mahmood, S., Qureshi, S., Memon, A.M., Zaman, T., "MODLEACH: A Variant of LEACH for WSNs", in 2013 Eighth International Conference on Broadband and Wireless Computing, Communication and Applications, 158-163, (2013).

[6] Smaragdakis, G., Matta, I., Bestavros, A., "SEP: A stable election protocol for clustered heterogeneous wireless sensor networks", Second Int. Work. Sens. Actor Netw. Protoc. Appl. (SANPA 2004), 1-11, (2004).

[7] Muruganathan, S.D., Ma, D.C.F., Bhasin, R.I., Fapojuwo, A.O., "A centralized energy-efficient routing protocol for wireless sensor networks", IEEE Commun. Mag., 43(3): 8-13, (2005).

[8] Peng, Z., Yin, H., Dong, H., Li, H., “LEACH Protocol based Two-Level Clustering Algorithm,” Int. J. Hybrid Inf. Technol., 8(10), 15-26, (2015).

[9] Brand, H., Rego, S., Cardoso, R., Jr, J.C., Networks, C., "MH-LEACH : A Distributed Algorithm for Multi-Hop Communication in Wireless Sensor Networks", in ICN2014, 55-61, (2014).

[10] Yang, H., Xu, J., Wang, R., Qian, L., "Energy-Efficient Multi-hop Routing Algorithm Based on LEACH", 578-587, (2013).

[11] Sharma S., Choudhary, S., "Heterogeneous multi-hop LEACH routing protocol”, in 2014 International Conference on Green Computing Communication and Electrical Engineering (ICGCCEE), 1-6, (2014). 
[12] Biradar, R.V., Sawant, S.R., Mudholkar, R.R., Patil, V.C., "Inter-Intra Cluster Multihop-LEACH Routing In Self-Organizing Wireless Sensor Networks", (2015).

[13] Farooq, M.O., Dogar, A.B., Shah, G.A., "MR-LEACH: Multi-hop Routing with Low Energy Adaptive Clustering Hierarchy", Fourth International Conference on Sensor Technologies and Applications, 262-268, (2010).

[14] Souid, I., Ben Chikha, H., El Monser, M., Gasmi, S., Attia, R., "Multi-hop dynamic clustering LEACH protocol for large scale networks", 22nd International Conference on Software, Telecommunications and Computer Networks (SoftCOM), 144-148, (2014).

[15] Lindsey, S., Raghavendra, C.S., "PEGASIS: Power-efficient gathering in sensor information systems", in Proceedings, IEEE Aerospace Conference, 3, 3-1125-3-1130, (2001).

[16] Manjeshwar, A., Agrawal, D.P., “APTEEN: a hybrid protocol for efficient routing and comprehensive information retrieval in wireless", in Proceedings 16th International Parallel and Distributed Processing Symposium, 8, (2002).

[17] Manjeshwar, A., Agrawal, D.P., "TEEN: a routing protocol for enhanced efficiency in wireless sensor networks", in Proceedings 15th International Parallel and Distributed Processing Symposium. 20092015, (2001).

[18] Shrivastav, K., Kulat, K.D., "Energy efficient scalability of three level hexagonal heterogeneous broad transmission distance protocol (3L-HEXA-HTBTDP) for WSN-IoT networks", Int. J. Commun. Syst., no. August, p. e3809, (2018).

[19] Arjunan, S., Pothula, S., Ponnurangam, D., "F5N-based unequal clustering protocol (F5NUCP) for wireless sensor networks", Int. J. Commun. Syst., no. August, p. e3811, (2018).

[20] Nigam, G.K., Dabas, C., "ESO-LEACH: PSO based energy efficient clustering in LEACH”, J. King Saud Univ. - Comput. Inf. Sci., 1: 4-11, (2018).

[21] Al-Sodairi, S., Ouni, R., "Reliable and energy-efficient multi-hop LEACH-based clustering protocol for wireless sensor networks", Sustain. Comput. Informatics Syst., 20: 1-13, (2018).

[22] Moorthi, Thiagarajan, R., "Energy consumption and network connectivity based on Novel-LEACHPOS protocol networks", Computer Communications, 149: 90-98, (2020).

[23] Cui, Z., Cao, Y., Cai, X., Cai, J., Chen, J., "Optimal LEACH protocol with modified bat algorithm for big data sensing systems in Internet of Things", Journal of Parallel and Distributed Computing, 132: 217-229, (2019).

[24] Chandra, A., Parvin, M., "Quasi-dynamic Load Balanced Clustering Protocol for Energy Efficient Wireless Sensor Networks”, Wireless Pers Commun, 111: 1589-1605, (2020).

[25] Pal, R., Yadav, S., Karnwal, R. et al., "EEWC: energy-efficient weighted clustering method based on genetic algorithm for HWSNs", Complex Intell. Syst., (2020).

[26] Ihsan, A., Saghar, K., Fatima, T., Hasan, O., "Formal comparison of LEACH and its extensions", Computer Standards \& Interfaces, 62: 119-127,( 2019). 